\title{
The local strong and weak solutions to a generalized Novikov equation
}

Shaoyong Lai* and Meng Wu

${ }^{\text {"Correspondence: }}$

laishaoy@swufe.edu.cn

Department of Mathematics,

Southwestern University of Finance

and Economics, Chengdu, 610074, China

\begin{abstract}
A nonlinear partial differential equation, which includes the Novikov equation as a special case, is investigated. The well-posedness of local strong solutions for the equation in the Sobolev space $H^{5}(R)$ with $s>\frac{3}{2}$ is established. Although the $H^{1}$-norm of the solutions to the nonlinear model does not remain constant, the existence of its local weak solutions in the lower order Sobolev space $H^{s}(R)$ with $1 \leq s \leq \frac{3}{2}$ is established under the assumptions $u_{0} \in H^{5}$ and $\left\|u_{0 x}\right\|_{L}<\infty$.
\end{abstract}

MSC: 35Q35; 35Q51

Keywords: local strong solution; local weak solution; generalized Novikov equation

\section{Introduction}

Novikov [1] derived the integrable equation with cubic nonlinearities

$$
u_{t}-u_{t x x}+4 u^{2} u_{x}=3 u u_{x} u_{x x}+u^{2} u_{x x x}
$$

which has been investigated by many scholars. Grayshan [2] studied both the periodic and the non-periodic Cauchy problem for Eq. (1) and discussed continuity results for the data-to-solution map in the Sobolev spaces. A Galerkin-type approximation method was used in Himonas and Holliman's paper [3] to establish the well-posedness of Eq. (1) in the Sobolev space $H^{s}(R)$ with $s>\frac{3}{2}$ on both the line and the circle. Hone et al. [4] applied the scattering theory to find non-smooth explicit soliton solutions with multiple peaks for Eq. (1). This multiple peak property is common with the Camassa-Holm and Degasperis-Procesi equations (see [5-7]). A matrix Lax pair for Eq. (1) was acquired in [8, 9] and was shown to be related to a negative flow in the Sawada-Kotera hierarchy. Sufficient conditions on the initial data to guarantee the formation of singularities in finite time for Eq. (1) were given in Jiang and Li [10]. Mi and Mu [11] obtained many dynamic results for a modified Novikov equation with a peak solution. It is shown in $\mathrm{Ni}$ and Zhou [12] that the Novikov equation associated with the initial value is locally well-posed in Sobolev space $H^{s}$ with $s>\frac{3}{2}$ by using the abstract Kato theorem. Two results about the persistence properties of the strong solution for Eq. (1) are established in [12]. Tiglay [13] proved the local well-posedness for the periodic Cauchy problem of the Novikov equation in Sobolev space $H^{s}(R)$ with $s>\frac{5}{2}$. The orbit invariants are used to show the existence of a periodic global strong solution if the Sobolev index $s \geq 3$ and a sign condition holds. For analytic initial data, the existence and uniqueness of analytic solutions for Eq. (1) are obtained in [13]. Using the Littlewood-Paley decomposition and nonhomogeneous Besov

C) 2013 Lai and Wu; licensee Springer. This is an Open Access article distributed under the terms of the Creative Commons Attribution License (http://creativecommons.org/licenses/by/2.0), which permits unrestricted use, distribution, and reproduction in any medium, provided the original work is properly cited. 
spaces, Yan et al. [14] proved that Eq. (1) is locally well-posed in the Besov space under certain assumptions. For other methods to handle the Novikov equation and the related partial differential equations, the reader is referred to [15-24] and the references therein.

We note that the coefficients of the terms $u^{2} u_{x}, u u_{x} u_{x x}$ and $u^{2} u_{x x x}$ in the Novikov equation (1) are 4,3 and 1 , respectively. Namely, $4=3+1$. This guarantees that the conservation law of Eq. (1) holds

$$
\int_{R}\left(u^{2}(t, x)+u_{x}^{2}(t, x)\right) d x=\int_{R}\left(u^{2}(0, x)+u_{x}^{2}(0, x)\right) d x,
$$

which takes a key role in obtaining various dynamic properties in the previous works.

Motivated by the desire to extend parts of local well-posedness results in $[3,11,12]$, we study the following model:

$$
u_{t}-u_{t x x}+m u^{2} u_{x}=a u u_{x} u_{x x}+b u^{2} u_{x x x}
$$

where $m, a$ and $b>0$ are arbitrary constants. Clearly, letting $m=4, a=3$ and $b=1$, Eq. (2) becomes the Novikov equation (1).

The objective of this paper is to investigate Eq. (2). Since $m, a$ and $b>0$ are arbitrary constants, we do not have the result that the $H^{1}$ norm of the solution of Eq. (2) remains constant. We will apply the Kato theorem for abstract differential equations to prove the existence and uniqueness of local solutions for Eq. (2) subject to the initial value $u_{0}(x) \in$ $H^{s}(R)\left(s>\frac{3}{2}\right)$. In addition, the existence of local weak solutions for Eq. (2) is established in the lower-order Sobolev space $H^{s}(R)$ with $1 \leq s \leq \frac{3}{2}$ under the assumptions $u_{0} \in H^{s}(R)$ and $\left\|u_{0 x}\right\|_{L^{\infty}}<\infty$.

The rest of this paper is organized as follows. The main results are given in Section 2. The proof of a local well-posedness result is established in Section 3, while the existence of local weak solutions is proved in Section 4.

\section{Main results}

Firstly, we state some notations.

The space of all infinitely differentiable functions $\phi(t, x)$ with compact support in $[0,+\infty) \times R$ is denoted by $C_{0}^{\infty} \cdot L^{p}=L^{p}(R)(1 \leq p<+\infty)$ is the space of all measurable functions $h$ such that $\|h\|_{L^{p}}^{p}=\int_{R}|h(t, x)|^{p} d x<\infty$. We define $L^{\infty}=L^{\infty}(R)$ with the standard norm $\|h\|_{L^{\infty}}=\inf _{m(e)=0} \sup _{x \in R \backslash e}|h(t, x)|$. For any real number $s, H^{s}=H^{s}(R)$ denotes the Sobolev space with the norm defined by

$$
\|h\|_{H^{s}}=\left(\int_{R}\left(1+|\xi|^{2}\right)^{s}|\hat{h}(t, \xi)|^{2} d \xi\right)^{\frac{1}{2}}<\infty
$$

where $\hat{h}(t, \xi)=\int_{R} e^{-i x \xi} h(t, x) d x$.

For $T>0$ and nonnegative number $s, C\left([0, T) ; H^{s}(R)\right)$ denotes the Frechet space of all continuous $H^{s}$-valued functions on $[0, T)$. We set $\Lambda=\left(1-\partial_{x}^{2}\right)^{\frac{1}{2}}$. For simplicity, throughout this article, we let $c$ denote any positive constant which is independent of parameter $\varepsilon$.

Defining

$$
\phi(x)= \begin{cases}e^{\frac{1}{x^{2}-1}}, & |x|<1 \\ 0, & |x| \geq 1,\end{cases}
$$


and setting $\phi_{\varepsilon}(x)=\varepsilon^{-\frac{1}{4}} \phi\left(\varepsilon^{-\frac{1}{4}} x\right)$ with $0<\varepsilon<\frac{1}{4}$ and $u_{\varepsilon 0}=\phi_{\varepsilon} \star u_{0}$, we know that $u_{\varepsilon 0} \in C^{\infty}$ for any $u_{0} \in H^{s}, s>0$ (see [17]).

We consider the Cauchy problem for Eq. (2)

$$
\left\{\begin{array}{l}
u_{t}-u_{t x x}+m u^{2} u_{x}=a u u_{x} u_{x x}+b u^{2} u_{x x x} \\
u(0, x)=u_{0}(x)
\end{array}\right.
$$

which is equivalent to

$$
\left\{\begin{array}{l}
u_{t}+b u^{2} u_{x}=\Lambda^{-2}\left[(b-m) u^{2} u_{x}+\frac{a-6 b}{2}\left(u u_{x}^{2}\right)_{x}+\frac{2 b-a}{2} u_{x}^{3}\right] \\
u(0, x)=u_{0}(x)
\end{array}\right.
$$

Now, we give our main results for problem (3).

Theorem 1 Let $u_{0}(x) \in H^{s}(R)$ with $s>\frac{3}{2}$. Then the Cauchy problem (3) has a unique solution $u(t, x) \in C\left([0, T) ; H^{s}(R)\right) \cap C^{1}\left([0, T) ; H^{s-1}(R)\right)$, where $T>0$ depends on $\left\|u_{0}\right\|_{H^{s}(R)}$.

It follows from Theorem 1 that for each $\varepsilon$ satisfying $0<\varepsilon<\frac{1}{4}$, the Cauchy problem

$$
\left\{\begin{array}{l}
u_{t}-u_{t x x}+m u^{2} u_{x}=a u u_{x} u_{x x}+b u^{2} u_{x x x}, \\
u(0, x)=u_{\varepsilon 0}(x), \quad x \in R
\end{array}\right.
$$

has a unique solution $u_{\varepsilon}(t, x) \in C^{\infty}\left(\left[0, T_{\varepsilon}\right) ; H^{\infty}\right)$, in which $T_{\varepsilon}$ may depend on $\varepsilon$. However, we shall show that under certain assumptions, there exist two constants $c$ and $T>0$, both independent of $\varepsilon$, such that the solution of problem (5) satisfies $\left\|u_{\varepsilon x}\right\|_{L^{\infty}} \leq c$ for any $t \in$ $[0, T)$ and there exists a weak solution $u(t, x) \in L^{2}\left([0, T], H^{s}(R)\right)$ for problem (3). These results are summarized in the following two theorems.

Theorem 2 If $u_{0}(x) \in H^{s}(R)$ with $s \in\left[1, \frac{3}{2}\right]$ such that $\left\|u_{0 x}\right\|_{L^{\infty}}<\infty$. Let $u_{\varepsilon 0}$ be defined as in system (5). Then there exist two constants $c$ and $T>0$, which are independent of $\varepsilon$, such that the solution $u_{\varepsilon}$ of problem (5) satisfies $\left\|u_{\varepsilon x}\right\|_{L^{\infty}} \leq c$ for any $t \in[0, T)$.

Theorem 3 Suppose that $u_{0}(x) \in H^{s}$ with $1 \leq s \leq \frac{3}{2}$ and $\left\|u_{0 x}\right\|_{L^{\infty}}<\infty$. Then there exists a $T>0$ such that problem (3) has a weak solution $u(t, x) \in L^{2}\left([0, T], H^{s}(R)\right)$ in the sense of distribution and $u_{x} \in L^{\infty}([0, T] \times R)$.

\section{Proof of Theorem 1}

Consider the abstract quasi-linear evolution equation

$$
\frac{d v}{d t}+A(v) v=f(v), \quad t \geq 0 \quad \text { and } \quad v(0)=v_{0} .
$$

Let $X$ and $Y$ be Hilbert spaces such that $Y$ is continuously and densely embedded in $X$, and let $Q: Y \rightarrow X$ be a topological isomorphism. Let $L(Y, X)$ be the space of all bounded linear operators from $Y$ to $X$. If $X=Y$, we denote this space by $L(X)$. We state the following conditions in which $\rho_{1}, \rho_{2}, \rho_{3}$ and $\rho_{4}$ are constants depending only on $\max \left\{\|y\|_{Y},\|z\|_{Y}\right\}$. 
(I) $A(y) \in L(Y, X)$ for $y \in X$ with

$$
\|(A(y)-A(z)) w\|_{X} \leq \rho_{1}\|y-z\|_{X}\|w\|_{Y}, \quad y, z, w \in Y,
$$

and $A(y) \in G(X, 1, \beta)$ (i.e., $A(y)$ is quasi-m-accretive), uniformly on bounded sets in $Y$.

(II) $Q A(y) Q^{-1}=A(y)+B(y)$, where $B(y) \in L(X)$ is bounded, uniformly on bounded sets in $Y$. Moreover,

$$
\|(B(y)-B(z)) w\|_{X} \leq \rho_{2}\|y-z\|_{Y}\|w\|_{X}, \quad y, z \in Y, w \in X
$$

(III) $f: Y \rightarrow Y$ extends to a map from $X$ into $X$, is bounded on bounded sets in $Y$, and satisfies

$$
\begin{aligned}
& \|f(y)-f(z)\|_{Y} \leq \rho_{3}\|y-z\|_{Y}, \quad y, z \in Y, \\
& \|f(y)-f(z)\|_{X} \leq \rho_{4}\|y-z\|_{X}, \quad y, z \in X .
\end{aligned}
$$

Kato theorem (see [25]) Assume that (I), (II) and (III) hold. If $v_{0} \in Y$, there is a maximal $T>0$ depending only on $\left\|v_{0}\right\|_{Y}$ and a unique solution $v$ to problem (6) such that

$$
v=v\left(\cdot, v_{0}\right) \in C([0, T) ; Y) \cap C^{1}([0, T) ; X) .
$$

Moreover, the map $v_{0} \rightarrow v\left(\cdot, v_{0}\right)$ is a continuous map from $Y$ to the space

$$
C([0, T) ; Y) \cap C^{1}([0, T) ; X) .
$$

We set $A(u)=b u^{2} \partial_{x}$ with constant $b>0, Y=H^{s}(R), X=H^{s-1}(R), \Lambda=\left(1-\partial_{x}^{2}\right)^{\frac{1}{2}}, f(u)=$ $\Lambda^{-2}\left[(b-m) u^{2} u_{x}+\frac{a-6 b}{2}\left(u u_{x}^{2}\right)_{x}+\frac{2 b-a}{2} u_{x}^{3}\right]$ and $Q=\Lambda^{s}$. We know that $Q$ is an isomorphism of $H^{s}$ onto $H^{s-1}$. In order to prove Theorem 1 , we only need to check that $A(u)$ and $f(u)$ satisfy assumptions (I)-(III).

Lemma 3.1 The operator $A(u)=b u^{2} \partial_{x}$ with $u \in H^{s}(R), s>\frac{3}{2}$ belongs to $G\left(H^{s-1}(R), 1, \beta\right)$.

Lemma 3.2 Let $A(u)=b u^{2} \partial_{x}$ with $u \in H^{s}(R)$ and $s>\frac{3}{2}$. Then $A(u) \in L\left(H^{s}(R), H^{s-1}(R)\right)$ for all $u \in H^{s}(R)$. Moreover,

$$
\|(A(u)-A(z)) w\|_{H^{s-1}} \leq \rho_{1}\|u-z\|_{H^{s-1}}\|w\|_{H^{s}}, \quad u, z, w \in H^{s}(R) .
$$

Lemma 3.3 For $s>\frac{3}{2}, u, z \in H^{s}(R)$ and $w \in H^{s-1}$, it holds that $B(u)=\left[\Lambda^{s}, b u^{2} \partial_{x}\right] \Lambda^{-s} \in$ $L\left(H^{s-1}\right)$ for $u \in H^{s}$ and

$$
\|(B(u)-B(z)) w\|_{H^{s-1}} \leq \rho_{2}\|u-z\|_{H^{s}}\|w\|_{H^{s-1}}
$$

The above three lemmas can be found in Ni and Zhou [12].

Lemma 3.4 Let $r$ and $q$ be real numbers such that $-r<q \leq r$. Then

$$
\begin{aligned}
& \|u v\|_{H^{q}} \leq c\|u\|_{H^{r}}\|v\|_{H^{q}} \quad \text { if } r>\frac{1}{2}, \\
& \|u v\|_{H^{r+q-1 / 2}} \leq c\|u\|_{H^{r}}\|v\|_{H^{q}} \quad \text { if } r<\frac{1}{2} .
\end{aligned}
$$


This lemma can be found in $[25,26]$.

Lemma 3.5 Let $u, z \in H^{s}$ with $s>\frac{3}{2}$ and $f(u)=\Lambda^{-2}\left[(b-m) u^{2} u_{x}+\frac{a-6 b}{2}\left(u u_{x}^{2}\right)_{x}+\frac{2 b-a}{2} u_{x}^{3}\right]$. Then $f$ is bounded on bounded sets in $H^{s}$ and satisfies

$$
\begin{aligned}
& \|f(u)-f(z)\|_{H^{s}} \leq \rho_{3}\|u-z\|_{H^{s}}, \\
& \|f(u)-f(z)\|_{H^{s-1}} \leq \rho_{4}\|u-z\|_{H^{s-1}} .
\end{aligned}
$$

Proof Using the algebra property of the space $H^{s_{0}}(R)$ with $s_{0}>\frac{1}{2}$, we get

$$
\begin{aligned}
& \|f(u)-f(z)\|_{H^{s}} \\
& \leq c\left(\left\|u^{3}-z^{3}\right\|_{H^{s-1}}+\left\|u u_{x}^{2}-z z_{x}^{2}\right\|_{H^{s-1}}+\left\|u_{x}^{3}-z_{x}^{3}\right\|_{H^{s-1}}\right) \\
& \leq c\left(\|u-z\|_{H^{s}}\left[\|u\|_{H^{s}}^{2}+\|u\|_{H^{s}}\|z\|_{H^{s}}+\|z\|_{H^{s}}^{2}\right]\right. \\
& \quad+\left\|u\left(u_{x}^{2}-z_{x}^{2}\right)+(u-z) z_{x}^{2}\right\|_{H^{s-1}} \\
& \left.\quad+\left\|u_{x}-z_{x}\right\|_{H^{s-1}}\left[\left\|u_{x}^{2}\right\|_{H^{s-1}}+\left\|u_{x}\right\|_{H^{s-1}}\|z\|_{H^{s-1}}+\|z\|_{H^{s-1}}^{2}\right]\right) \\
& \leq \rho_{3}\|u-z\|_{H^{s}},
\end{aligned}
$$

which completes the proof of (9). Using $s-1>\frac{1}{2}$ and the first inequality in Lemma 3.4, we have

$$
\begin{aligned}
& \left\|u u_{x}^{2}-z z_{x}^{2}\right\|_{H^{s-2}} \\
& \quad \leq\left\|u\left(u_{x}^{2}-z_{x}^{2}\right)+z_{x}^{2}(u-z)\right\|_{H^{s-2}} \\
& \quad \leq\left\|u\left(u_{x}+z_{x}\right)\left(u_{x}-z_{x}\right)\right\|_{H^{s-2}}+\|u-z\|_{s-1}\left\|z_{x}^{2}\right\|_{H^{s-2}} \\
& \quad \leq c\left\|u\left(u_{x}+z_{z}\right)\right\|_{H^{s-1}}\|u-z\|_{H^{s-1}}+\|u-z\|_{s^{-1}}\left\|z_{x}\right\|_{H^{s-1}}^{2} \\
& \quad \leq c\|u-z\|_{H^{s-1}}\left(\|u\|_{H^{s}}^{2}+\|z\|_{H^{s}}^{2}\right)
\end{aligned}
$$

and

$$
\begin{aligned}
& \left\|u_{x}^{3}-z_{x}^{3}\right\|_{H^{s-2}} \\
& \quad \leq\left\|\left(u_{x}-z_{x}\right)\left(u_{x}^{2}+u_{x} z_{x}+z_{x}^{2}\right)\right\|_{H^{s-2}} \\
& \quad \leq c\left\|u_{x}-z_{x}\right\|_{H^{s-2}}\left\|u_{x}^{2}+u_{x} z_{x}+z_{x}^{2}\right\|_{H^{s-1}} \\
& \quad \leq c\|u-z\|_{H^{s-1}}\left(\|u\|_{H^{s}}^{2}+\|z\|_{H^{s}}^{2}\right) .
\end{aligned}
$$

Using (12) and (13) yields

$$
\begin{aligned}
& \|f(u)-f(z)\|_{H^{s-1}} \\
& \quad \leq c\left(\left\|u^{3}-z^{3}\right\|_{H^{s-2}}+\left\|u u_{x}^{2}-z z_{x}^{2}\right\|_{H^{s-2}}+\left\|u_{x}^{3}-z_{x}^{3}\right\|_{H^{s-2}}\right) \\
& \quad \leq c\|u-z\|_{H^{s-1}}\left(1+\|u\|_{H^{s-1}}^{2}+\|z\|_{H^{s-1}}^{2}+\|u\|_{H^{s}}^{2}+\|z\|_{H^{s}}^{2}\right),
\end{aligned}
$$

which completes the proof of inequality (10). 
Proof of Theorem 1 Using the Kato theorem, Lemmas 3.1, 3.2, 3.3 and Lemma 3.5, we know that system (3) or problem (4) has a unique solution

$$
u(t, x) \in C\left([0, T) ; H^{s}(R)\right) \cap C^{1}\left([0, T) ; H^{s-1}(R)\right) .
$$

\section{Proofs of Theorems 2 and 3}

Using the first equation of system (3) derives

$$
\frac{d}{d t} \int_{R}\left(u^{2}+u_{x}^{2}\right) d x+2(a-3 b) \int_{R} u u_{x}^{3} d x=0
$$

from which we have the conservation law

$$
\int_{R}\left(u^{2}+u_{x}^{2}\right) d x+2(a-3 b) \int_{0}^{t} \int_{R} u u_{x}^{3} d x=\int_{R}\left(u_{0}^{2}+u_{0 x}^{2}\right) d x
$$

Lemma 4.1 (Kato and Ponce [26]) If $r \geq 0$, then $H^{r} \cap L^{\infty}$ is an algebra. Moreover,

$$
\|u v\|_{r} \leq c\left(\|u\|_{L^{\infty}}\|v\|_{r}+\|u\|_{r}\|v\|_{L^{\infty}}\right)
$$

where $c$ is a constant depending only on $r$.

Lemma 4.2 (Kato and Ponce [26]) Let $r>0$. If $u \in H^{r} \cap W^{1, \infty}$ and $v \in H^{r-1} \cap L^{\infty}$, then

$$
\left\|\left[\Lambda^{r}, u\right] v\right\|_{L^{2}} \leq c\left(\left\|\partial_{x} u\right\|_{L^{\infty}}\left\|\Lambda^{r-1} v\right\|_{L^{2}}+\left\|\Lambda^{r} u\right\|_{L^{2}}\|v\|_{L^{\infty}}\right)
$$

Lemma 4.3 Let $s>\frac{3}{2}$ and the function $u(t, x)$ is a solution of problem (3) and the initial data $u_{0}(x) \in H^{s}(R)$. Then the following results hold:

$$
\|u\|_{H^{1}} \leq\left\|u_{0}\right\|_{H^{1}(R)} e^{\frac{|a-3 b|}{2} \int_{0}^{t}\left\|u_{x}\right\|_{L^{\infty}(R)}^{2} d \tau}
$$

For $q \in(0, s-1]$, there is a constant $c$ only depending on $m, a$ and $b$ such that

$$
\begin{aligned}
& \int_{R}\left(\Lambda^{q+1} u\right)^{2} d x \\
& \quad \leq \int_{R}\left[\left(\Lambda^{q+1} u_{0}\right)^{2}\right] d x+c \int_{0}^{t}\|u\|_{H^{q+1}}^{2}\left(\left\|u_{x}\right\|_{L^{\infty}}\|u\|_{L^{\infty}}+\left\|u_{x}\right\|_{L^{\infty}}^{2}\right) d \tau
\end{aligned}
$$

For $q \in[0, s-1]$, there is a constant $c$ only depending on $m, a$ and $b$ such that

$$
\left\|u_{t}\right\|_{H^{q}} \leq c\|u\|_{H^{q+1}}\left(\|u\|_{L^{\infty}}\|u\|_{H^{1}}+\|u\|_{L^{\infty}}\left\|u_{x}\right\|_{L^{\infty}}+\left\|u_{x}\right\|_{L^{\infty}}^{2}\right) .
$$

Proof Using $\left|2 u u_{x}\right| \leq\left(u^{2}+u_{x}^{2}\right)$, the Gronwall inequality and (15) derives (16).

Using $\partial_{x}^{2}=-\Lambda^{2}+1$ and the Parseval equality gives rise to

$$
\int_{R} \Lambda^{q} u \Lambda^{q} \partial_{x}^{3}\left(u^{3}\right) d x=-3 \int_{R}\left(\Lambda^{q+1} u\right) \Lambda^{q+1}\left(u^{2} u_{x}\right) d x+3 \int_{R}\left(\Lambda^{q} u\right) \Lambda^{q}\left(u^{2} u_{x}\right) d x
$$


For $q \in(0, s-1]$, applying $\left(\Lambda^{q} u\right) \Lambda^{q}$ to both sides of the first equation of system (3) and integrating with respect to $x$ by parts, we have the identity

$$
\begin{aligned}
\frac{1}{2} \frac{d}{d t} \int_{R}\left(\left(\Lambda^{q} u\right)^{2}+\left(\Lambda^{q} u_{x}\right)^{2}\right) d x= & -(m-b) \int_{R}\left(\Lambda^{q} u\right) \Lambda^{q}\left(u^{2} u_{x}\right) d x \\
& -b \int_{R}\left(\Lambda^{q+1} u\right) \Lambda^{q+1}\left(u^{2} u_{x}\right) d x-2 b \int_{R} \Lambda^{q} u \Lambda^{q} u_{x}^{3} d x \\
& +(a-6 b) \int_{R} \Lambda^{q} u \Lambda^{q}\left(u u_{x} u_{x x}\right) d x
\end{aligned}
$$

We will estimate the terms on the right-hand side of (19) separately. For the first term, by using the Cauchy-Schwarz inequality and Lemmas 4.1 and 4.2, we have

$$
\begin{aligned}
\left|\int_{R}\left(\Lambda^{q} u\right) \Lambda^{q}\left(u^{2} u_{x}\right) d x\right|= & \left|\int_{R}\left(\Lambda^{q} u\right)\left[\Lambda^{q}\left(u^{2} u_{x}\right)-u^{2} \Lambda^{q} u_{x}\right] d x+\int_{R}\left(\Lambda^{q} u\right) u^{2} \Lambda^{q} u_{x} d x\right| \\
\leq & c\|u\|_{H^{q}}\left(2\|u\|_{L^{\infty}}\left\|u_{x}\right\|_{L^{\infty}}\|u\|_{H^{q}}+\left\|u_{x}\right\|_{L^{\infty}}\|u\|_{L^{\infty}}\|u\|_{H^{q}}\right) \\
& +\|u\|_{L^{\infty}}\left\|u_{x}\right\|_{L^{\infty}}\left\|\Lambda^{q} u\right\|_{L^{2}}^{2} \\
\leq & c\|u\|_{H^{q}}^{2}\|u\|_{L^{\infty}}\left\|u_{x}\right\|_{L^{\infty}} .
\end{aligned}
$$

Using the above estimate to the second term yields

$$
\left|\int_{R}\left(\Lambda^{q+1} u\right) \Lambda^{q+1}\left(u^{2} u_{x}\right) d x\right| \leq c\|u\|_{H^{q+1}}^{2}\|u\|_{L^{\infty}}\left\|u_{x}\right\|_{L^{\infty}} .
$$

Using the Cauchy-Schwarz inequality and Lemma 4.1, we obtain

$$
\begin{aligned}
\left|\int_{R}\left(\Lambda^{q} u_{x}\right) \Lambda^{q}\left(u u_{x}^{2}\right) d x\right| & \leq\left\|\Lambda^{q} u_{x}\right\|_{L^{2}}\left\|\Lambda^{q}\left(u u_{x}^{2}\right)\right\|_{L^{2}} \\
& \leq c\|u\|_{H^{q+1}}\left(\|u\|_{L^{\infty}}\left\|u_{x}^{2}\right\|_{H^{q}}+\|u\|_{H^{q}}\left\|u_{x}^{2}\right\|_{L^{\infty}}\right) \\
& \leq c\|u\|_{H^{q+1}}^{2}\left(\|u\|_{L^{\infty}}\left\|u_{x}\right\|_{L^{\infty}}+\left\|u_{x}\right\|_{L^{\infty}}^{2}\right) .
\end{aligned}
$$

For the last term in (19), using $u\left(u_{x}^{2}\right)_{x}=\left(u u_{x}^{2}\right)_{x}-u_{x} u_{x}^{2}$ results in

$$
\begin{aligned}
\left|\int_{R}\left(\Lambda^{q} u\right) \Lambda^{q}\left(u u_{x} u_{x x}\right) d x\right| & \leq \frac{1}{2}\left|\int_{R} \Lambda^{q} u_{x} \Lambda^{q}\left(u u_{x}^{2}\right) d x\right|+\frac{1}{2}\left|\int_{R} \Lambda^{q} u \Lambda^{q}\left[u_{x} u_{x}^{2}\right] d x\right| \\
& =K_{1}+K_{2} .
\end{aligned}
$$

For $K_{1}$, it follows from (22) that

$$
K_{1} \leq c\|u\|_{H^{q+1}}^{2}\left(\|u\|_{L^{\infty}}\left\|u_{x}\right\|_{L^{\infty}}+\left\|u_{x}\right\|_{L^{\infty}}^{2}\right)
$$

For $K_{2}$, applying Lemma 4.1 derives

$$
\begin{aligned}
K_{2} & \leq c\|u\|_{H^{q}}\left\|u_{x} u_{x}^{2}\right\|_{H^{q}} \\
& \leq c\|u\|_{H^{q}}\left(\left\|u_{x}\right\|_{L^{\infty}}\left\|u_{x}^{2}\right\|_{H^{q}}+\left\|u_{x}\right\|_{H^{q}}\left\|u_{x}^{2}\right\|_{L^{\infty}}\right) \\
& \leq c\|u\|_{H^{q+1}}^{2}\left\|u_{x}\right\|_{L^{\infty}}^{2} .
\end{aligned}
$$


It follows from (20)-(25) that there exists a constant $c$ such that

$$
\begin{aligned}
& \frac{1}{2} \frac{d}{d t} \int_{R}\left[\left(\Lambda^{q} u\right)^{2}+\left(\Lambda^{q} u_{x}\right)^{2}\right] d x \\
& \quad \leq c\|u\|_{H^{q+1}}^{2}\left(\left\|u_{x}\right\|_{L^{\infty}}\|u\|_{L^{\infty}}+\left\|u_{x}\right\|_{L^{\infty}}^{2}\right)
\end{aligned}
$$

Integrating both sides of the above inequality with respect to $t$ results in inequality (17).

To estimate the norm of $u_{t}$, we apply the operator $\left(1-\partial_{x}^{2}\right)^{-1}$ to both sides of the first equation of system (3) to obtain the equation

$$
u_{t}=\left(1-\partial_{x}^{2}\right)^{-1}\left[-\frac{m}{3}\left(u^{3}\right)_{x}+\frac{b}{3} \partial_{x}^{3}\left(u^{3}\right)-2 b \partial_{x}\left(u u_{x}^{2}\right)+(a-2 b) u u_{x} u_{x x}\right]
$$

Applying $\left(\Lambda^{q} u_{t}\right) \Lambda^{q}$ to both sides of Eq. (27) for $q \in[0, s-1]$ gives rise to

$$
\begin{aligned}
& \int_{R}\left(\Lambda^{q} u_{t}\right)^{2} d x \\
& \quad=\int_{R}\left(\Lambda^{q} u_{t}\right) \Lambda^{q-2}\left[\partial_{x}\left(-\frac{m}{3} u^{3}+\frac{b}{3} \partial_{x}^{2} u^{3}-2 b u u_{x}^{2}\right)+(a-2 b) u u_{x} u_{x x}\right] d \tau .
\end{aligned}
$$

For the right-hand of Eq. (28), we have

$$
\begin{aligned}
& \left|\int_{R}\left(\Lambda^{q} u_{t}\right)\left(1-\partial_{x}^{2}\right)^{-1} \Lambda^{q} \partial_{x}\left(-\frac{m}{3} u^{3}-2 b u u_{x}^{2}\right) d x\right| \\
& \quad \leq c\left\|u_{t}\right\|_{H^{q}}\left(\int_{R}\left(1+\xi^{2}\right)^{q-1}\left[\int\left[-\frac{m}{3} \widehat{u^{2}}(\xi-\eta) \widehat{u}(\eta)-2 b \widehat{u u_{x}}(\xi-\eta) \widehat{u_{x}}(\eta)\right] d \eta\right]^{2}\right)^{\frac{1}{2}} \\
& \quad \leq c\left\|u_{t}\right\|_{H^{q}}\|u\|_{H^{1}}\|u\|_{H^{q+1}}\|u\|_{L^{\infty}} .
\end{aligned}
$$

Since

$$
\begin{aligned}
\int\left(\Lambda^{q} u_{t}\right)\left(1-\partial_{x}^{2}\right)^{-1} \Lambda^{q} \partial_{x}^{2}\left(u^{2} u_{x}\right) d x= & -\int\left(\Lambda^{q} u_{t}\right) \Lambda^{q}\left(u^{2} u_{x}\right) d x \\
& +\int\left(\Lambda^{q} u_{t}\right)\left(1-\partial_{x}^{2}\right)^{-1} \Lambda^{q}\left(u^{2} u_{x}\right) d x
\end{aligned}
$$

using Lemma 4.1, $\left\|u^{2} u_{x}\right\|_{H^{q}} \leq c\left\|\left(u^{3}\right)_{x}\right\|_{H^{q}} \leq c\|u\|_{L^{\infty}}^{2}\|u\|_{H^{q+1}}$ and $\|u\|_{L^{\infty}} \leq\|u\|_{H^{1}}$, we have

$$
\begin{aligned}
& \left|\int\left(\Lambda^{q} u_{t}\right) \Lambda^{q}\left(u^{2} u_{x}\right) d x\right| \\
& \quad \leq c\left\|u_{t}\right\|_{H^{q}}\left\|u^{2} u_{x}\right\|_{H^{q}} \\
& \quad \leq c\left\|u_{t}\right\|_{H^{q}}\|u\|_{L^{\infty}}\|u\|_{H^{1}}\|u\|_{H^{q+1}}
\end{aligned}
$$

and

$$
\left|\int\left(\Lambda^{q} u_{t}\right)\left(1-\partial_{x}^{2}\right)^{-1} \Lambda^{q}\left(u^{2} u_{x}\right) d x\right| \leq c\left\|u_{t}\right\|_{H^{q}}\|u\|_{L^{\infty}}\|u\|_{H^{1}}\|u\|_{H^{q+1}}
$$


Using the Cauchy-Schwarz inequality and Lemma 4.1 yields

$$
\begin{aligned}
& \left|\int_{R}\left(\Lambda^{q} u_{t}\right)\left(1-\partial_{x}^{2}\right)^{-1} \Lambda^{q}\left(u u_{x} u_{x x}\right) d x\right| \\
& \quad \leq c\left\|u_{t}\right\|_{H^{q}}\left\|u u_{x} u_{x x}\right\|_{H^{q-2}} \leq c\left\|u_{t}\right\|_{H^{q}}\left\|u\left(u_{x}^{2}\right)_{x}\right\|_{H^{q-2}} \\
& \quad \leq c\left\|u_{t}\right\|_{H^{q}}\left\|\left[u\left(u_{x}^{2}\right)\right]_{x}-(u)_{x} u_{x}^{2}\right\|_{H^{q-2}} \\
& \quad \leq c\left\|u_{t}\right\|_{H^{q}}\left(\left\|u u_{x}^{2}\right\|_{H^{q-1}}+\left\|u_{x} u_{x}^{2}\right\|_{H^{q-2}}\right) \\
& \quad \leq c\left\|u_{t}\right\|_{H^{q}}\left(\left\|u u_{x}^{2}\right\|_{H^{q}}+\left\|u_{x} u_{x}^{2}\right\|_{H^{q}}\right) \\
& \quad \leq c\left\|u_{t}\right\|_{H^{q}}\|u\|_{H^{q+1}}\left(\|u\|_{L^{\infty}}\left\|u_{x}\right\|_{L^{\infty}}+\left\|u_{x}\right\|_{L^{\infty}}^{2}\right) .
\end{aligned}
$$

Applying (29)-(33) into (28) yields the inequality

$$
\left\|u_{t}\right\|_{H^{q}} \leq c\|u\|_{H^{q+1}}\left(\|u\|_{L^{\infty}}\|u\|_{H^{1}}+\|u\|_{L^{\infty}}\left\|u_{x}\right\|_{L^{\infty}}+\left\|u_{x}\right\|_{L^{\infty}}^{2}\right)
$$

for a constant $c>0$. This completes the proof of Lemma 4.3.

Lemma 4.4 ([17]) For $s>0, u_{0} \in H^{s}(R)$ and $u_{\varepsilon 0}=\phi_{\varepsilon} \star u_{0}$, the following estimates hold for any $\varepsilon$ with $0<\varepsilon<\frac{1}{4}$

$$
\begin{aligned}
& \left\|u_{\varepsilon 0 x}\right\|_{L^{\infty}} \leq c\left\|u_{0 x}\right\|_{L^{\infty}} \quad \text { and } \quad\left\|u_{\varepsilon 0}\right\|_{H^{q}} \leq c, \quad \text { if } q \leq s \\
& \left\|u_{\varepsilon 0}\right\|_{H^{q}} \leq c \varepsilon^{\frac{s-q}{4}}, \quad \text { if } q>s, \\
& \left\|u_{\varepsilon 0}-u_{0}\right\|_{H^{q}} \leq c \varepsilon^{\frac{s-q}{4}}, \quad \text { if } q \leq s \\
& \left\|u_{\varepsilon 0}-u_{0}\right\|_{H^{s}}=o(1),
\end{aligned}
$$

where $c$ is a constant independent of $\varepsilon$.

Proof of Theorem 2 Using notation $u=u_{\varepsilon}$ and differentiating both sides of the first equation of problem (5) or Eq. (27) with respect to $x$ give rise to

$$
\begin{aligned}
u_{t x} & +\frac{a-2 b}{2} u u_{x}^{2}+b u^{2} u_{x x} \\
& =\frac{m-b}{3} u^{3}+\Lambda^{-2}\left[\frac{b-m}{3} u^{3}+\frac{a-6 b}{2} u u_{x}^{2}+\frac{2 b-a}{2}\left(u_{x}^{3}\right)_{x}\right] .
\end{aligned}
$$

Letting $p>0$ be an integer and multiplying the above equation by $\left(u_{x}\right)^{2 p+1}$ and then integrating the resulting equation with respect to $x$ yield the equality

$$
\begin{aligned}
& \frac{1}{2 p+2} \frac{d}{d t} \int_{R}\left(u_{x}\right)^{2 p+2} d x+\frac{(a-2 b)(p+1)-2 b}{2 p+2} \int_{R} u\left(u_{x}\right)^{2 p+3} d x \\
& =\frac{m-b}{3} \int_{R} u^{3}\left(u_{x}\right)^{2 p+1} d x \\
& \quad+\int_{R}\left(u_{x}\right)^{2 p+1} \Lambda^{-2}\left[\frac{b-m}{3} u^{3}+\frac{a-6 b}{2} u u_{x}^{2}+\frac{2 b-a}{2}\left(u_{x}^{3}\right)_{x}\right] d x .
\end{aligned}
$$


Applying the Hölder's inequality yields

$$
\begin{aligned}
& \frac{1}{2 p+2} \frac{d}{d t} \int_{R}\left(u_{x}\right)^{2 p+2} d x \\
& \leq\left\{\frac{|m-b|}{3}\left(\int_{R}\left|u^{3}\right|^{2 p+2} d x\right)^{\frac{1}{2 p+2}}+\left(\int_{R}|G|^{2 p+2} d x\right)^{\frac{1}{2 p+2}}\right\}\left(\int_{R}\left|u_{x}\right|^{2 p+2} d x\right)^{\frac{2 p+1}{2 p+2}} \\
& \quad+\left|\frac{(a-2 b)(p+1)-2 b}{2 p+2}\right|\left\|u_{x}\right\|_{L^{\infty}}\|u\|_{L^{\infty}} \int_{R}\left|u_{x}\right|^{2 p+2} d x
\end{aligned}
$$

or

$$
\begin{aligned}
& \frac{d}{d t}\left(\int_{R}\left(u_{x}\right)^{2 p+2} d x\right)^{\frac{1}{2 p+2}} \\
& \leq \leq\left\{\frac{|m-b|}{3}\left(\int_{R}\left|u^{3}\right|^{2 p+2} d x\right)^{\frac{1}{2 p+2}}+\left(\int_{R}|G|^{2 p+2} d x\right)^{\frac{1}{2 p+2}}\right\} \\
& \quad+\left|\frac{(a-2 b)(p+1)-2 b}{2 p+2}\right|\left\|u_{x}\right\|_{L^{\infty}}\|u\|_{L^{\infty}}\left(\int_{R}\left|u_{x}\right|^{2 p+2} d x\right)^{\frac{1}{2 p+2}},
\end{aligned}
$$

where

$$
G=\Lambda^{-2}\left[\frac{b-m}{3} u^{3}+\frac{a-6 b}{2} u u_{x}^{2}+\frac{2 b-a}{2}\left(u_{x}^{3}\right)_{x}\right] .
$$

Since $\|f\|_{L^{p}} \rightarrow\|f\|_{L^{\infty}}$ as $p \rightarrow \infty$ for any $f \in L^{\infty} \cap L^{2}$, integrating both sides of the inequality (42) with respect to $t$ and taking the limit as $p \rightarrow \infty$ result in the estimate

$$
\left\|u_{x}\right\|_{L^{\infty}} \leq\left\|u_{0 x}\right\|_{L^{\infty}}+c \int_{0}^{t}\left[\|u\|_{L^{\infty}}^{3}+\|G\|_{L^{\infty}}+\frac{|a-2 b|}{2}\|u\|_{L^{\infty}}\left\|u_{x}\right\|_{L^{\infty}}^{2}\right] d \tau,
$$

where $c$ only depends on $m, a, b$.

Using the algebraic property of $H^{s_{0}}(R)$ with $s_{0}>\frac{1}{2}$ and the inequality (16) yields

$$
\|u\|_{L^{\infty}} \leq\|u\|_{H^{1}} \leq\left\|u_{0}\right\|_{H^{1}} e^{c \int_{0}^{t}\left\|u_{x}\right\|^{2} d \tau}
$$

and

$$
\begin{aligned}
\|G\|_{L^{\infty}} & \leq c\|G\|_{H^{\frac{1}{2}+}} \\
& =c\left\|\Lambda^{-2}\left[\frac{b-m}{3} u^{3}+\frac{a-6 b}{2} u u_{x}^{2}+\frac{2 b-a}{2}\left(u_{x}^{3}\right)_{x}\right]\right\|_{H^{\frac{1}{2}+}} \\
& \leq c\left(\left\|u^{3}\right\|_{H^{0}}+\left\|u u_{x}^{2}\right\|_{H^{0}}+\left\|u_{x}^{3}\right\|_{H^{0}}\right) \\
& \leq c\left(\|u\|_{H^{1}}^{3}+\|u\|_{H^{1}}\left(1+\left\|u_{x}\right\|_{L^{\infty}}^{2}\right)\right) \\
& \leq c e^{c \int_{0}^{t}\left\|u_{x}\right\|^{2} d \tau}\left(1+\left\|u_{x}\right\|_{L^{\infty}}^{2}\right),
\end{aligned}
$$

where $c$ is a constant independent of $\varepsilon$. From (45), we have

$$
\int_{0}^{t}\|G\|_{L^{\infty}} d \tau \leq c \int_{0}^{t} e^{c \int_{0}^{\tau}\left\|u_{x}\right\|^{2} d \zeta}\left(1+\left\|u_{x}\right\|_{L^{\infty}}^{2}\right) d \tau
$$


It follows from (43) and (46) that

$$
\left\|u_{x}\right\|_{L^{\infty}} \leq\left\|u_{0 x}\right\|_{L^{\infty}}+c \int_{0}^{t}\left[e^{c \int_{0}^{\tau}\left\|u_{x}\right\|^{2} d \zeta}\left(1+\left\|u_{x}\right\|_{L^{\infty}}^{2}\right)+1+\left\|u_{x}\right\|_{L^{\infty}}^{2}\right] d \tau .
$$

It follows from the contraction mapping principle that there is a $T>0$ such that the equation

$$
\|W\|_{L^{\infty}}=\left\|u_{0 x}\right\|_{L^{\infty}}+c \int_{0}^{t}\left[\left(1+\|W\|_{L^{\infty}}^{2}\right) \exp \left(c \int_{0}^{\tau}\|W\|_{L^{\infty}}^{2} d \varsigma\right)+1+\|W\|_{L^{\infty}}^{2}\right] d \tau
$$

has a unique solution $W \in C[0, T]$. Using the theorem presented on p.51 in $\mathrm{Li}$ and Olver [18] yields that there are constants $T>0$ and $c>0$, which are independent of $\varepsilon$, such that $\left\|u_{x}\right\|_{L^{\infty}} \leq W(t)$ for arbitrary $t \in[0, T]$, which leads to the conclusion of Theorem 2 .

Using Theorem 2, (17), (18) and (44), notation $u_{\varepsilon}=u$ and Gronwall's inequality results in the inequalities

$$
\left\|u_{\varepsilon}\right\|_{H^{q}} \leq c \exp \left[\int_{0}^{t}\left(\left\|u_{\varepsilon x}\right\|_{L^{\infty}}\left\|u_{\varepsilon}\right\|_{L^{\infty}}+\left\|u_{\varepsilon x}\right\|_{L^{\infty}}^{2}\right) d \tau\right] \leq c
$$

and

$$
\left\|u_{\varepsilon t}\right\|_{H^{r}} \leq\left\|u_{\varepsilon}\right\|_{H^{r+1}}\left(\left\|u_{\varepsilon}\right\|_{L^{\infty}}\left\|u_{\varepsilon}\right\|_{H^{1}}+\left\|u_{\varepsilon}\right\|_{L^{\infty}}\left\|u_{\varepsilon x}\right\|_{L^{\infty}}+\left\|u_{\varepsilon x}\right\|_{L^{\infty}}^{2}\right) \leq c
$$

where $q \in(0, s], r \in(0, s-1]$ and $t \in[0, T)$. It follows from Aubin's compactness theorem that there is a subsequence of $\left\{u_{\varepsilon}\right\}$, denoted by $\left\{u_{\varepsilon_{n}}\right\}$, such that $\left\{u_{\varepsilon_{n}}\right\}$ and their temporal derivatives $\left\{u_{\varepsilon_{n} t}\right\}$ are weakly convergent to a function $u(t, x)$ and its derivative $u_{t}$ in $L^{2}\left([0, T], H^{s}\right)$ and $L^{2}\left([0, T], H^{s-1}\right)$, respectively. Moreover, for any real number $R_{1}>0,\left\{u_{\varepsilon_{n}}\right\}$ is convergent to the function $u$ strongly in the space $L^{2}\left([0, T], H^{q}\left(-R_{1}, R_{1}\right)\right)$ for $q \in(0, s]$ and $\left\{u_{\varepsilon_{n} t}\right\}$ converges to $u_{t}$ strongly in the space $L^{2}\left([0, T], H^{r}\left(-R_{1}, R_{1}\right)\right)$ for $r \in[0, s-1]$.

Proof of Theorem 3 From Theorem 2, we know that $\left\{u_{\varepsilon_{n} x}\right\}\left(\varepsilon_{n} \rightarrow 0\right)$ is bounded in the space $L^{\infty}$. Thus, the sequences $\left\{u_{\varepsilon_{n}}\right\},\left\{u_{\varepsilon_{n} x}\right\},\left\{u_{\varepsilon_{n} x}^{2}\right\}$ and $\left\{u_{\varepsilon_{n} x}^{3}\right\}$ are weakly convergent to $u$, $u_{x}, u_{x}^{2}$ and $u_{x}^{3}$ in $L^{2}\left([0, T], H^{r}\left(-R_{1}, R_{1}\right)\right)$ for any $r \in[0, s-1)$, separately. Hence, $u$ satisfies the equation

$$
\begin{aligned}
& -\int_{0}^{T} \int_{R} u\left(g_{t}-g_{x x t}\right) d x d t \\
& =\int_{0}^{T} \int_{R}\left(\frac{m}{3} u^{3} g_{x}-\frac{b}{3} u^{3} g_{x x x}+\frac{a-2 b}{2} u_{x}^{3} g-\frac{a-6 b}{2} u u_{x}^{2} g_{x}\right) d x d t
\end{aligned}
$$

with $u(0, x)=u_{0}(x)$ and $g \in C_{0}^{\infty}$. Since $X=L^{1}([0, T] \times R)$ is a separable Banach space and $\left\{u_{\varepsilon_{n} x}\right\}$ is a bounded sequence in the dual space $X^{*}=L^{\infty}([0, T] \times R)$ of $X$, there exists a subsequence of $\left\{u_{\varepsilon_{n} x}\right\}$, still denoted by $\left\{u_{\varepsilon_{n} x}\right\}$, weakly star convergent to a function $v$ in $L^{\infty}([0, T] \times R)$. As $\left\{u_{\varepsilon_{n} x}\right\}$ weakly converges to $u_{x}$ in $L^{2}([0, T] \times R)$, it results that $u_{x}=v$ almost everywhere. Thus, we obtain $u_{x} \in L^{\infty}([0, T] \times R)$. 


\section{Competing interests}

The authors declare that they have no competing interests.

\section{Authors' contributions}

The article is a joint work of two authors who contributed equally to the final version of the paper. All authors read and approved the final manuscript.

\section{Acknowledgements}

Thanks are given to referees whose comments and suggestions are very helpful to revise our paper. This work is supported by both the Fundamental Research Funds for the Central Universities (JBK120504) and the Applied and Basic Project of Sichuan Province (2012JY0020)

\section{Received: 8 December 2012 Accepted: 3 May 2013 Published: 20 May 2013}

\section{References}

1. Novikov, V: Generalizations of the Camassa-Holm equation. J. Phys. A, Math. Theor. 42, 342002 (2009)

2. Grayshan, K: Peakon solutions of the Novikov equation and properties of the data-to-solution map. J. Math. Anal. Appl. 397, 515-521 (2013)

3. Himonas, A, Holliman, C: The Cauchy problem for the Novikov equation. Nonlinearity 25, 449-479 (2012)

4. Hone, AN, Lundmark, H, Szmigielski, J: Explicit multipeakon solutions of Novikov's cubically nonlinear integrable Camassa-Holm type equation. Dyn. Partial Differ. Equ. 6, 253-289 (2009)

5. Camassa, R, Holm, D: An integrable shallow water equation with peaked solitons. Phys. Rev. Lett. 71, 1661-1664 (1993)

6. Constantin, A, Lannes, D: The hydro-dynamical relevance of the Camassa-Holm and Degasperis-Procesi equations. Arch. Ration. Mech. Anal. 193, 165-186 (2009)

7. Constantin, J, Escher, J: Wave breaking for nonlinear nonlocal shallow water equations. Acta Math. 181, 229-243 (1998)

8. Hone, AN, Wang, JP: Integrable peakon equations with cubic nonlinearity. J. Phys. A 41, 372002-10 (2008)

9. Himonas, A, Misiolek, G, Ponce, G, Zhou, Y: Persistence properties and unique continuation of solutions of Camassa-Holm equation. Commun. Math. Phys. 271, 511-522 (2007)

10. Jiang, ZH, Ni, LD: Blow-up phenomenon for the integrable Novikov equation. J. Math. Anal. Appl. 385, $551-558$ (2012)

11. Mi, YS, Mu, CL: On the Cauchy problem for the modified Novikov equation with peakon solutions. J. Differ. Equ. 254, 961-982 (2013)

12. Ni, L, Zhou, Y: Well-posedness and persistence properties for the Novikov equation. J. Differ. Equ. 250, 3002-3021 (2011)

13. Tiglay, F: The periodic Cauchy problem for Novikov's equation. Math. AP (2010). arXiv:1009.1820v1

14. Yan, W, Li, YS, Zhang, YM: The Cauchy problem for the integrable Novikov equation. J. Differ. Equ. 253, 298-318 (2012)

15. Yan, W, Li, YS, Zhang, YM: Global existence and blow-up phenomena for the weakly dissipative Novikov equation. Nonlinear Anal. 75, 2464-2473 (2012)

16. Lai, SY, Li, N, Wu, YH: The existence of global strong and weak solutions for the Novikov equation. J. Math. Anal. Appl. 399, 682-691 (2013)

17. Lai, SY, Wu, YH: The local well-posedness and existence of weak solutions for a generalized Camassa-Holm equation. J. Differ. Equ. 248, 2038-2063 (2010)

18. Li, Y, Olver, P: Well-posedness and blow-up solutions for an integrable nonlinearly dispersive model wave equation. J. Differ. Equ. 162, 27-63 (2000)

19. Mohajer, K, Szmigielski, J: On an inverse problem associated with an integrable equation of Camassa-Holm type: explicit formulas on the real axis. Inverse Probl. 28, 015002 (2012)

20. Yin, ZY: On the Cauchy problem for an intergrable equation with peakon solutions. III. J. Math. 47, 649-666 (2003)

21. Zhao, L, Zhou, SG: Symbolic analysis and exact travelling wave solutions to a new modified Novikov equation. Appl. Math. Comput. 217, 590-598 (2010)

22. Zhou, Y: Blow-up solutions to the DGH equation. J. Funct. Anal. 250, 227-248 (2007)

23. Faramarz, T, Mohammad, S: Existence and blow up of solutions to a Petrovsky equation with memory and nonlinear source. Bound. Value Probl. 2012, Article ID 50 (2012)

24. Zhang, Y, Liu, DM, Mu, CL, Zheng, P: Blow-up for an evolution p-Laplace system with nonlocal sources and inner absorptions. Bound. Value Probl. 2011, Article ID 29 (2011)

25. Kato, T: Quasi-linear equations of evolution with applications to partial differential equations. In: Spectral Theory and Differential Equations. Lecture Notes in Math., vol. 448, pp. 25-70. Springer, Berlin (1975)

26. Kato, T, Ponce, G: Commutator estimates and the Euler and Navier-Stokes equations. Commun. Pure Appl. Math. 41, 891-907 (1998)

doi:10.1186/1687-2770-2013-134

Cite this article as: Lai and Wu: The local strong and weak solutions to a generalized Novikov equation. Boundary Value Problems 2013 2013:134. 